Article

\title{
New Results on Controllability for a Class of Fractional Integrodifferential Dynamical Systems with Delay in Banach Spaces ${ }^{\dagger}$
}

\author{
Daliang Zhao
}

Citation: Zhao, D. New Results on Controllability for a Class of

Fractional Integrodifferential Dynamical Systems with Delay in Banach Spaces. Fractal Fract. 2021, 5, 89. https://doi.org/10.3390/ fractalfract5030089

Academic Editors: Ivanka Stamova Gani Stamov and Xiaodi Li

Received: 9 July 2021

Accepted: 2 August 2021

Published: 4 August 2021

Publisher's Note: MDPI stays neutral with regard to jurisdictional claims in published maps and institutional affiliations.

Copyright: (C) 2021 by the author. Licensee MDPI, Basel, Switzerland. This article is an open access article distributed under the terms and conditions of the Creative Commons Attribution (CC BY) license (https:/ / creativecommons.org/licenses/by/ $4.0 /)$.
School of Mathematics and Statistics, Shandong Normal University, Jinan 250014, China; dlzhao928@sdnu.edu.cn + Research supported by the National Natural Science Foundation of China under grant 62073202, and a project of Shandong Province Higher Educational Science and Technology Program of China under the grant J18KA233.

\begin{abstract}
The present work addresses some new controllability results for a class of fractional integrodifferential dynamical systems with a delay in Banach spaces. Under the new definition of controllability, first introduced by us, we obtain some sufficient conditions of controllability for the considered dynamic systems. To conquer the difficulties arising from time delay, we also introduce a suitable delay item in a special complete space. In this work, a nonlinear item is not assumed to have Lipschitz continuity or other growth hypotheses compared with most existing articles. Our main tools are resolvent operator theory and fixed point theory. At last, an example is presented to explain our abstract conclusions.
\end{abstract}

Keywords: fractional integrodifferential dynamical systems; delay; controllability; resolvent operator; fixed point theory

\section{Introduction}

The purpose of this work is to investigate the controllability of the following fractional integrodifferential dynamical systems with a delay in Banach spaces:

$$
\left\{\begin{array}{l}
{ }^{C} D^{r} z(t)=\mathcal{A} z(t)+g\left(t, z_{t}, H_{z}(t)\right)+\mathcal{B} x(t), t \in V:=[0, T], \\
z(t)=\psi(t), \quad t \in[-c, 0],
\end{array}\right.
$$

where the state variable $z(\cdot)$ takes values in Banach space $E .{ }^{C} D^{r}$ denotes the Caputo derivative with order $r \in(0,1) . \mathcal{A}: D(\mathcal{A}) \subset E \rightarrow E$ is a closed linear unbounded operator on $E . x$ is a control function defined in $L^{2}(V ; U)$, where $U$ is a Banach space. $\mathcal{B}: U \rightarrow E$ is a bounded linear operator. $\psi(t) \in C([-c, 0] ; E) . g$ is a given nonlinear item satisfying some appropriate hypotheses and

$$
H_{z}(t)=\int_{0}^{t} h\left(t, s, z_{s}\right) d s
$$

with $h: \Delta \times L([-c, 0] ; E) \rightarrow E$ where $\Delta=\{(t, s) \in V \times V: s \leq t\} . L([-c, 0] ; E)$ is the space of $E$-valued Bochner integrable functions on $[-c, 0]$ with the norm $\|z\|_{L[-c, 0]}=$ $\int_{-c}^{0}\|z(t)\| d t$

The theory of fractional calculus has a long-standing history, and has received considerable attention due mainly to its potential and wide applications in various fields, such as viscoelasticity, signal processing, pure mathematics, control, electromagnetics, etc. (see [1-7]). In the modeling of many phenomena in various science and technology fields, fractional differential equations, including both ordinary and partial ones, are considered to be more powerful tools than their corresponding integer-order counterparts. Many phenomena, such as electronics, fluid dynamics, biological models and chemical kinetics, 
cannot be described through classical differential equations; in these cases, integrodifferential equations play an important role in describing most of these natural phenomena. For more details of fractional calculus theory, and the results of integrodifferential equations, one can see [8-14], and the references therein.

Time delay occurs frequently and is inevitable in various practical systems of the real world [15-19]. This is especially true for dynamical evolution processes which are closely related to time. Hence, if we intend to accurately describe the evolution systems, we must consider the effect of time delay. With the development of the applications for fractional calculus, research into the controllability of fractional dynamical systems with delay is increasingly extensive [20-25].

It is well known that control theory is an interdisciplinary subject involving economics, engineering and mathematics, which investigates and analyses some dynamical behaviors of various systems [26-32]. It is worth noting that controllability is of importance in some research fields of networks such as logical control networks, and steady-state design of large-dimensional Boolean networks. Logical control networks are widely used in controllability, evolutionary games, stability and optimal control, and many fundamental results have been established for them [33-35]. With the rapid development of control theory, the problem of controllability for a special kind of logical control networks, Boolean control networks, was also investigated by researchers. For more details of the recent works in this regard, we refer readers to [36-38]. Controllability is one of the fundamental concepts in mathematical control theory. On the one hand, in the study of controllability for fractional dynamical systems, the hypothesis of noncompact semigroups is especially important, as the compactness of the semigroups is only applicable in finite-dimensional spaces, since the inverse of control operator cannot be ensured if the state space is infinitedimensional. Some technical errors caused by the compactness of semigroups have been pointed out by Hernández et al. [39]. On the other hand, how to introduce the mild solutions in infinite dimensional spaces is another particularly important step. For example, Hernández et al. [40] also pointed out that the definition of mild solutions in some articles, such as $[23,41]$, was inappropriate because it was only a simple extension of the integerorder mild solutions. We know that a fractional evolution dynamical system is usually transformed into a form of Volterra integral equation to obtain its mild solutions. Therefore, the theory of resolvent operators is a powerful tool to study such systems. Compared with the mild solutions constructed by some probability density functions (El-Borai [42]), it is found that in the investigation of evolution dynamical systems with unbounded operators in infinite dimensional spaces, resolvent operators seem to be more appropriate since they are direct generalizations of $C_{0}$-semigroups and cosine families. This is why we adopted the resolvent operator theory to define mild solutions and investigate the controllability of the considered fractional dynamical systems in this paper.

Some excellent results concerning the controllability of various nonlinear fractional dynamical systems were obtained. However, most of these controllability problems were investigated under the hypothesis that the nonlinear item $f$ is Lipschitz continuous, compact or satisfies some other growth conditions, see [20-23,43,44] for example. We point out that, as a more stronger smooth condition than continuity, Lipschitz continuity, is only regarded as an idealized supposition in many cases, which is difficult to apply to practical problems. Furthermore, there are scarcely any results on the controllability of fractional integrodifferential dynamical systems with delay, except for [20,22-24]. However, in [20], the authors still supposed the nonlocal item to be Lipschitz continuous, and that the nonlinear function satisfied certain growth conditions. Notice that in [22-24], authors hobtained controllability results for fractional delay differential and integrodifferential dynamical systems with the nonlinear functions also being Lipschitz continuous. Therefore, a very natural question is whether the considered fractional integrodifferential dynamical systems with delay are controllable when the nonlinear item is only continuous, rather than Lipschitz continuous. This is also the main motivation for the present work. 
Compared with the above-mentioned research, the main contributions of this work are as below: (i) Under the new definition of controllability, we suppose that the nonlinear function here only has continuity rather than Lipschitz continuity and other certain growth assumptions. (ii) In order to overcome the obstacles caused by time delay, we utilize a special complete space $L([-c, 0] ; E)$ in which to define the suitable time delay item $z_{t}$.

The organization of the rest of this work is as follows. Some necessary preparations are given in Section 2. In Section 3, sufficient conditions of the controllability for system (1) are obtained. An example is provided in Section 4 to illustrate the effectiveness of the abstract results.

\section{Preliminaries}

Notation 1. Let $R$ denote the set of real numbers, $R^{+}$the set of positive numbers. $\Gamma$ is the gamma function. I represents the identity operator. Suppose $E$ to be a Banach space along with the norm $\|\cdot\|$. $\mathcal{B}: U \rightarrow E$ is a bounded linear operator where $U$ is also a Banach space. $\mathcal{A}: D(\mathcal{A}) \subset E \rightarrow E$ is a closed linear unbounded operator on $E$. Denoted by $\mathcal{D}$, the dense domain of closed linear unbounded operator $\mathcal{A}$ equipped with the graph norm $\|z\|_{\mathcal{D}}=\|z\|+\|\mathcal{A} z\| ; C(V ; E)$ stands for the space of E-valued continuous functions on $V$ with the norm $\|\cdot\|_{C(V ; E)} \cdot C([-C, T] ; E)$ denotes the Banach space of continuous functions from $[-c, T]$ to $E$ with the usual supreme norm. Denote the norm of the space $C([-c, 0] ; E)$ by $\|\cdot\|_{c}$ for brevity. $C^{r}(V ; E), r \in(0,1)$, represents the space formed of all the $r$-Hölder E-valued continuous functions from $V$ into $E$ equipped with the norm $\|z\|_{C^{r}(V ; E)}=\|z\|_{C(V ; E)}+[|z|]_{C^{r}(V ; E)}$, where $[|z|]_{C^{r}(V ; E)}=\sup _{t, s \in V, t \neq s} \frac{\|z(t)-z(s)\|}{(t-s)^{r}}$. Assume that $J \subset R$, and $1 \leq p \leq \infty$. For measurable function $z: J \rightarrow R$, define the norm

$$
\|z\|_{L^{p}(J)}= \begin{cases}\left(\int_{J}|z(t)|^{p} d t\right)^{\frac{1}{p}}, & 1 \leq p<\infty, \\ \inf _{\mu(\bar{J}=0)}\left\{\sup _{t \in J-\bar{J}}|z(t)|\right\}, & p=\infty\end{cases}
$$

where $\mu(\bar{J})$ is the Lebesgue measure on $\bar{J}$. Let $L^{p}(J, R)$ be the Banach space of all Lebesgue functions $z: J \rightarrow R$ with $\|\cdot\|_{L^{p}(J)}<\infty$. The space of bounded linear operators from $E$ into Banach space $F$ is defined as $\mathcal{L}(E, F)$ provided with the operator norm $\|\cdot\|_{\mathcal{L}(E, F)}$, and $\mathcal{L}(E, E)$ is written as $\mathcal{L}(E)$ with norm $\|\cdot\|_{\mathcal{L}(E)}$.

To deal with the inconveniences caused by delay during the investigation of controllability in the sequel, we utilize a special complete space $L([-c, 0] ; E)$. For $z \in C(V ; E)$ and $t \in V$, define a function $z_{t}$ :

$$
z_{t}(\theta)= \begin{cases}z(t+\theta), & t+\theta \geq 0 \\ \psi(t+\theta), & t+\theta \leq 0\end{cases}
$$

for any $\theta \in[-c, 0]$. It is not difficult to deduce that $z_{t} \in L([-c, 0] ; E)$.

The basic definitions of fractional calculus are presented as follows. For further details, please see [11] and the references therein.

The Riemann-Liouville fractional integral of order $r>0$ and the lower limit zero for a continuous function $u$ is given by

$$
I_{0^{+}}^{r} u(t)=\frac{1}{\Gamma(r)} \int_{0}^{t}(t-s)^{r-1} u(s) d s, t>0,
$$

if the right side integral is pointwise defined on $(0,+\infty)$. 
The Riemann-Liouville derivative and the lower limit zero for a continuous function $u:(0, \infty) \rightarrow R$ is defined as

$$
{ }^{L} D_{0^{+}}^{r} u(t)=\frac{1}{\Gamma(n-r)} \frac{d^{n}}{d t^{n}} \int_{0}^{t} \frac{u(s)}{(t-s)^{r-n+1}} d s, t>0, n-1<r<n, r>0,
$$

and the corresponding Caputo fractional derivative of order $r>0$ with the lower limit zero for a continuous function $u:(0, \infty) \rightarrow R$ is given by

$$
{ }^{C} D_{0^{+}}^{r} u(t)={ }^{L} D_{0^{+}}^{r}\left(u(t)-\sum_{k=0}^{n-1} \frac{u^{k}(0)}{k !} t^{k}\right), t>0, n-1<r<n, r>0 .
$$

It is noted that if $u(t) \in C^{n}[0, \infty)$, then one can obtain

$$
{ }^{C} D_{0^{+}}^{r} u(t)=\frac{1}{\Gamma(n-r)} \int_{0}^{t} \frac{u^{(n)}(s)}{(t-s)^{r-n+1}} d s=I^{n-r} u^{(n)}(t), t>0, n-1<r<n, r>0 .
$$

Throughout this paper, we suppose that the following integral equation

$$
z(t)=\frac{1}{\Gamma(r)} \int_{0}^{t} \frac{\mathcal{A} z(s)}{(t-s)^{1-r}} d s, t \geq 0
$$

has an associated resolvent operator $\{\mathcal{N}(t)\}_{t \geq 0}$ on $E$.

Definition 1 ([45]). Bounded linear operator $\{\mathcal{N}(t)\}_{t \geq 0} \subset \mathcal{L}(E)$ is defined as a resolvent operator for (3) if the following assumptions are satisfied:

(I) $\mathcal{N}(t)$ is strongly continuous on $R^{+}$and $\mathcal{N}(0)=I$;

(II) $\mathcal{N}(t) \mathcal{D} \subset \mathcal{D}, \mathcal{A N}(t) z=\mathcal{N}(t) \mathcal{A} z$ for all $z \in \mathcal{D}$ and every $t \geq 0$;

(III) $\mathcal{N}(t) z=z+\frac{1}{\Gamma(r)} \int_{0}^{t} \frac{\mathcal{A} z(s)}{(t-s)^{1-r}} d s, z \in \mathcal{D}, t \geq 0$.

Definition 2 ([45]). A resolvent operator $\mathcal{N}(t)$ for (3) is called analytic, if the function $\mathcal{N}(\cdot)$ : $R^{+} \rightarrow \mathcal{L}(E)$ admits an analytic extension to a sector $\sum\left(0, \theta_{0}\right)=\left\{\lambda \in C:|\arg (\lambda)|<\theta_{0}\right\}$ for some $0<\theta_{0} \leq \frac{\pi}{2}$.

Definition $3([45]) . z \in C(V ; E)$ is defined as a mild solution to the Volterra integral equation

$$
z(t)=k(t)+\frac{1}{\Gamma(r)} \int_{0}^{t} \frac{\mathcal{A} z(s)}{(t-s)^{1-r}} d s, t \in V
$$

where $k \in L^{1}(V ; E)$, if $\int_{0}^{t} \frac{z(s)}{(t-s)^{1-r}} d s \in \mathcal{D}$ for all $t \in V$ and

$$
z(t)=k(t)+\frac{1}{\Gamma(r)} \mathcal{A} \int_{0}^{t} \frac{z(s)}{(t-s)^{1-r}} d s
$$

holds for $V$.

Lemma 1 ([45]). Suppose $\mathcal{N}(t)$ is an analytic resolvent operator of $(4)$ and $k \in C^{r}(V ; E), k(0)=$ 0. Then

$$
z(t)=\mathcal{N}(t) k(t)+\int_{0}^{t} \dot{\mathcal{N}}(t-s)[k(s)-k(t)] d s, \quad t \in V,
$$

is a mild solution of (4) and $z \in C^{r}(V ; E)$.

Lemma 2 (Mönch). Assume that $D$ is a closed and convex subset of a Banach space $E$ and $z_{0} \in D$. Suppose that the continuous operator $\mathcal{A}: D \rightarrow D$ satisfies: $C \subset D$ countable, $C \subset$ $\overline{\mathrm{CO}}\left(\left\{z_{0}\right\} \cup \mathcal{A}(C)\right) \rightarrow C$ is relatively compact. Then, $\mathcal{A}$ has a fixed point in $D$. 
Lemma 3 (Hölder Inequality). Assume that $p_{1}, p_{2} \geq 1$, and $\frac{1}{p_{1}}+\frac{1}{p_{2}}=1$. If $z_{1} \in L^{p_{1}}(J, R), z_{2} \in$ $L^{p_{2}}(J, R)$, then, $z_{1} z_{2} \in L^{1}(J, R)$ and $\left\|z_{1} z_{2}\right\|_{L^{1} J} \leq\left\|z_{1}\right\|_{L^{p_{1} J}}\left\|z_{2}\right\|_{L^{p_{2} J}}$.

The theory of Kuratowski's measures of noncompactness is crucial to the following proof work. For more details, see [46].

Lemma 4. Suppose $E$ to be a Banach space and $\zeta(\cdot)$ to be the Kuratowski's measures of noncompactness. (1) Let $D_{1}, D_{2}$ be bounded sets of $E$ and $\lambda \in R$. Then

(i) $\zeta\left(D_{1}\right)=0$ if, and only if, $D_{1}$ is relatively compact;

(ii) $\zeta\left(D_{1}\right) \leq \zeta\left(D_{2}\right)$ if $D_{1} \subset D_{2}$;

(iii) $\zeta\left(\lambda D_{1}\right)=|\lambda| \zeta\left(D_{1}\right)$;

(iv) $\zeta\left(D_{1}+D_{2}\right) \leq \zeta\left(D_{1}\right)+\zeta\left(D_{2}\right)$

(v) $\zeta\left(x_{0} \cup D_{1}\right)=\zeta\left(D_{1}\right), x_{0} \in E$;

(2) Let $D \subset C(V ; E)$ be bounded. Then, $D(t)$ is bounded in $E$ and $\zeta(D(t)) \leq \zeta(D)$.

(3) Let $D \subset C(V ; E)$ be bounded and equicontinuous. Then, $\zeta(D(t))$ is continuous on $V$, and $\zeta(D)=\max _{t \in V} \zeta(D(t))$.

(4) Let $D=\left\{u_{n}\right\} \subset C(V ; E)$ be countable. If there exists $\Phi \in L^{1}(W)$ such that $\left\|u_{n}(t)\right\| \leq$ $\Phi(t)$ a.e.t $\in W, n=1,2, \cdots$, then $\zeta(D(t))$ is integrable on $V$, and

$$
\zeta\left(\left\{\int_{V} u_{n}(t) d t: n \in N\right\}\right) \leq 2 \int_{V} \zeta(D(t)) d t .
$$

On the premise of no confusion, Kuratowski's measures of noncompactness of a bounded subset in spaces $E, C(V ; E)$ and $L([-c, 0] ; E)$ are all denoted by $\zeta(\cdot)$.

Finally, we introduce some important results:

Lemma 5. If $z_{n}$ converges to $z_{0}$ in $C(V ; E)$ as $n \rightarrow+\infty$, then one has that $\left(z_{n}\right)_{t}$ converges to $\left(z_{0}\right)_{t}$ in $L([-c, 0]$; E) for each $t \in V$ as $n \rightarrow+\infty$.

Proof. By means of (2), we have

$$
\left\|\left(z_{n}\right)_{t}-\left(z_{0}\right)_{t}\right\|_{L[-c, 0]}= \begin{cases}\int_{0}^{t}\left\|z_{n}(s)-z_{0}(s)\right\| d s, \quad t \leq c, \\ \int_{t-c}^{t}\left\|z_{n}(s)-z_{0}(s)\right\| d s, \quad t \geq c .\end{cases}
$$

This indicates that $\left\|\left(z_{n}\right)_{t}-\left(z_{0}\right)_{t}\right\|_{L[-c, 0]} \leq c\left\|z_{n}-z_{0}\right\|_{C(V ; E)}$. This completes the proof.

On the basis of the definition of Kuratowski's measures of noncompactness and Lemma 5, it is not difficult to obtain:

Lemma 6. Assume that $\left\{z_{n}\right\}_{n=1}^{\infty}$ is a bounded countable sequence in $C(V ; E)$. Then one can obtain

$$
\zeta\left(\left\{\left(z_{n}\right)_{t}\right\}\right) \leq c \zeta\left(\left\{z_{n}\right\}\right), \forall t \in V .
$$

\section{Main Results}

On the basis of the Riemann-Liouville fractional integral, together with Definition 3, the mild solution to system (1) can be obtained as follows: 
Definition 4. For each $x \in L^{2}(V ; U)$, a function $z \in C(W ; E)$ is said to be a mild solution of fractional dynamical system (1) on $W$, if $\int_{0}^{t} \frac{z(s)}{(t-s)^{1-r}} d s \in \mathcal{D}$ for all $t \in\left[0, T_{0}\right]$ and $z$ satisfies the following integral equation

$z(t)=\left\{\begin{array}{l}\psi(0)+\frac{1}{\Gamma(r)} \mathcal{A} \int_{0}^{t} \frac{z(s)}{(t-s)^{1-r}} d s+\frac{1}{\Gamma(r)} \int_{0}^{t} \frac{g\left(s, z_{s}, H_{z}(s)\right)}{(t-s)^{1-r}} d s+\frac{1}{\Gamma(r)} \int_{0}^{t} \frac{\mathcal{B} x(s)}{(t-s)^{1-r}} d s, \quad t \in\left[0, T_{0}\right], \\ \psi(t), \quad t \in[-c, 0],\end{array}\right.$ where $W=\left[-c, T_{0}\right], T_{0} \in(0, T]$.

Definition 5. The system (1) is said to be controllable on $V=[0, T]$, if, for every $\psi(t) \in$ $C([-c, 0] ; E)$ and $z_{1} \in E$, there exists a control $x \in L^{2}(V, U)$ and a constant $T_{0} \in(0, T]$, such that a mild solution $z$ of system (1) on $W=\left[-c, T_{0}\right]$ satisfies $z\left(T_{0}\right)=z_{1}$.

Remark 1. Compared with the existing definitions in $[20,21,43,44,47]$, etc., in which $z_{1}$ is obtained at the right endpoint $T$, the present definition, which we introduced with $z_{1}$ arriving at $T_{0} \in(0, T]$, is weaker.

Next, we impose the main hypotheses on the components of the systems:

Hypothesis $\mathbf{1}$ (H1). $g: V \times L([-c, 0] ; E) \times E \rightarrow E$ is continuous and takes bounded sets in $V \times L([-c, 0] ; E) \times E$ into bounded sets in $E$.

Hypothesis 2 (H2). $h: \Delta \times L([-c, 0] ; E) \rightarrow E$ is continuous where $\Delta=\{(t, s) \in V \times V:$ $s \leq t\}$.

Hypothesis 3 (H3). (i) The linear operator $\mathcal{B}: L^{2}(V ; U) \rightarrow L^{1}(V ; E)$ is bounded, and there exists a constant $Q_{1}>0$ such that $\|\mathcal{B}\|_{\mathcal{L}(U, E)} \leq Q_{1}$;

(ii) The linear operator $\Lambda(t)$ defined by

$$
\Lambda(t) x=\mathcal{N}(t) \mathcal{B}_{x}(t)+\int_{0}^{t} \dot{\mathcal{N}}(t-s)\left(\mathcal{B}_{x}(s)-\mathcal{B}_{x}(t)\right) d s
$$

where $\mathcal{B}_{x}(t)=\frac{1}{\Gamma(r)} \int_{0}^{t} \frac{\mathcal{B} x(s)}{(t-s)^{1-r}} d s$, has an invertible operator $\Lambda^{-1}(t)$ takeing values in $L^{2}(V ; U) / \operatorname{ker} \Lambda(t)$ for each $t \in V$, and there exists a constant $Q_{2}>0$ such that $\sup \left\|\Lambda^{-1}(\cdot)\right\|_{\mathcal{L}\left(E ; L^{2}(V ; U) / \operatorname{ker} \Lambda(\cdot)\right)} \leq Q_{2}$.

Hypothesis 4 (H4). (i) There exist constants $r_{i} \in(0, r)$ and real-valued functions $k_{i} \in L^{\frac{1}{r_{i}}}\left(V ; R^{+}\right)$, $i=1,2$, such that for any bounded subsets $D_{1} \subset L([-c, 0] ; E), D_{2} \subset E$,

$$
\zeta\left(g\left(t, D_{1}, D_{2}\right)\right) \leq k_{1}(t) \zeta\left(D_{1}\right)+k_{2}(t) \zeta\left(D_{2}\right), t \in V .
$$

(ii) There exists a function $l \in L^{1}\left(V ; R^{+}\right)$such that, for any bounded subset $D \subset L([-c, 0]$;E),

$$
\zeta(h(t, s, D)) \leq l(s) \zeta(D),(t, s) \in \Delta .
$$

(iii) There exists a constant $l_{0}>0$ such that

$$
\zeta\left(\Lambda^{-1}(\cdot)(D)(t)\right) \leq l_{0} \zeta(D), t \in V
$$

for any bounded set $D \subset E$.

We point out that resolvent operator $\{\mathcal{N}(t)\}_{t \geq 0}$ is supposed to be analytic in the rest of this work. In light of [45], we can assume that $N_{1}, N_{2}$ are positive numbers, such that 
$\|\dot{\mathcal{N}}(t)\|_{\mathcal{L}(E)} \leq N_{1} t^{-1},\|\ddot{\mathcal{N}}(t)\|_{\mathcal{L}(E)} \leq N_{2} t^{-2}$ for all $t \in(0, T] ; N_{0}$ is a positive constant, such that $\|\mathcal{N}(t)\|_{\mathcal{L}(E)} \leq N_{0}$ for all $t \in V$.

For simplicity, take

$$
\mathcal{G}_{z}(t)=\frac{1}{\Gamma(r)} \int_{0}^{t} \frac{g\left(s, z_{s}, H_{z}(s)\right)}{(t-s)^{1-r}} d s
$$

and let

$$
\begin{gathered}
\theta_{i}=\frac{1-r_{i}}{r-r_{i}}, \quad \vartheta_{i}=\frac{r-1}{1-r_{i}}, \quad i=1,2 ; \\
K_{1}=2 c \theta_{1}^{1-r_{1}}\left\|k_{1}\right\|_{L^{\frac{1}{r_{1}}}}+4 c \theta_{2}^{1-r_{2}}\left\|k_{2}\right\|_{L^{\frac{1}{r_{2}}}}\|l\|_{L^{1}} ; \\
K_{2}=2 c \theta_{1}\left\|k_{1}\right\|_{L^{\frac{1}{r_{1}}}}+4 c \theta_{2}\left\|k_{2}\right\|_{L^{\frac{1}{r_{2}}}}\|l\|_{L^{1}} ;
\end{gathered}
$$

and

$$
Q=N_{0} K_{1}+4 N_{1}\left(K_{1}+K_{2}\right) .
$$

In addition, for the purpose of simplifying our next work, we provide the next two necessary conclusions:

Lemma 7. (i) Assume that $g\left(\cdot, z ., H_{z}(\cdot)\right): V \times L([-c, 0] ; E) \times E \rightarrow E$ is continuous. Then, $\mathcal{G}_{z} \in C^{r}(V ; E)$ and $\left[\left|\mathcal{G}_{z}\right|\right]_{C^{r}} \leq \frac{2}{\Gamma(r+1)}\left\|g\left(\cdot, z ., H_{z}(\cdot)\right)\right\|_{C(V ; E)}$.

(ii) Assume that $x(\cdot) \in L^{2}(V ; U)$. Then, $\mathcal{B}_{x} \in C^{r}(V ; E)$ and $\left[\left|\mathcal{B}_{x}\right|\right]_{C^{r}} \leq \frac{2}{\Gamma(r+1)}\|\mathcal{B}\|_{\mathcal{L}(U, E)}$ $\|x(\cdot)\|_{L^{2}}$.

Proof. For $t \in[0, T)$ and $\iota>0$ such that $t+\iota \in V$, we have

$$
\begin{aligned}
\left\|\mathcal{G}_{z}(t+\iota)-\mathcal{G}_{z}(t)\right\| \leq & \frac{1}{\Gamma(r)} \int_{0}^{t}\left[\frac{1}{(t-s)^{1-r}}-\frac{1}{(t+\iota-s)^{1-r}}\right]\left\|g\left(s, z_{s}, H_{z}(s)\right)\right\| d s \\
& +\frac{1}{\Gamma(r)} \int_{t}^{t+\iota} \frac{\left\|g\left(s, z_{s}, H_{z}(s)\right)\right\|}{(t+\iota-s)^{1-r}} d s \\
\leq & \frac{1}{\Gamma(r)}\left\|g\left(\cdot, z,, H_{z}(\cdot)\right)\right\|_{C(V ; E)}\left(\frac{(t+\iota)^{r}-t^{r}+\iota^{r}}{r}+\frac{\iota^{r}}{r}\right) \\
\leq & \frac{2}{\Gamma(r+1)}\left\|g\left(\cdot, z, H_{z}(\cdot)\right)\right\|_{C(V ; E)^{l^{r}},}
\end{aligned}
$$

which implies that $\left[\left|\mathcal{G}_{z}\right|\right]_{C^{r}} \leq \frac{2}{\Gamma(r+1)}\left\|g\left(\cdot, z ., H_{z}(\cdot)\right)\right\|_{C(V ; E)}$ and $\mathcal{G}_{z} \in C^{r}(V ; E)$.

Repeating a similar process, we can obtain $\left[\left|\mathcal{B}_{x}\right|\right]_{C^{r}} \leq \frac{2}{\Gamma(r+1)}\|\mathcal{B}\|_{\mathcal{L}(U, E)}\|x(\cdot)\|_{L^{2}}$ and $\mathcal{B}_{x} \in C^{r}(V ; E)$. This completes the proof.

Lemma 8. Assume that (H1), (H2) and (H4) (i), (ii) hold. Then, operators $\mathcal{K}_{i}: C(V ; E) \rightarrow$ $C(V ; E)(i=1,2)$, defined by

$$
\begin{gathered}
\left(\mathcal{K}_{1} z\right)(t)=\int_{0}^{t}\left[(t-s)^{r-1}-\left(t_{1}-s\right)^{r-1}\right] g\left(s, z_{s}, H_{z}(s)\right) d s, t \in V, t<t_{1}, \\
\left(\mathcal{K}_{2} z\right)(t)=\int_{t}^{t_{1}}\left(t_{1}-s\right)^{r-1} g\left(s, z_{s}, H_{z}(s)\right) d s, t, t_{1} \in V, t<t_{1}
\end{gathered}
$$

satisfy $\zeta\left(\left(\mathcal{K}_{i} D\right)(t)\right) \leq K_{i}\left(t_{1}-t\right)^{r} \zeta(D)(i=1,2)$ for any countable bounded set $D \subset C(V ; E)$. 
Proof. Obviously, we can check that $\mathcal{K}_{i}(i=1,2)$ takes bounded sets in $C(V ; E)$ into bounded sets in $C(V ; E)$. Generally, a bounded countable set is chosen $D=\left\{z_{n}\right\}_{n=1}^{\infty}$. From Lemma 6, we have

$$
\zeta\left(\left\{\left(z_{n}\right)_{s}\right\}\right) \leq c \zeta\left(\left\{z_{n}\right\}\right) .
$$

By means of Lemma 3, Lemma 4 (4) and the well-known inequality

$$
t^{\varrho}-s^{\varrho} \geq(t-s)^{\varrho}, \varrho \in[1,+\infty), 0<s \leq t,
$$

one has

$$
\begin{aligned}
& \zeta\left(\left(\mathcal{K}_{1} D\right)(t)\right)=\zeta\left(\left\{\left(\mathcal{K}_{1} z_{n}\right)(t)\right\}\right) \\
& =\zeta\left(\left\{\int_{0}^{t}\left[(t-s)^{r-1}-\left(t_{1}-s\right)^{r-1}\right] g\left(s,\left(z_{n}\right)_{s}, H_{z_{n}}(s)\right) d s\right\}\right) \\
& \leq 2 \int_{0}^{t}\left[(t-s)^{r-1}-\left(t_{1}-s\right)^{r-1}\right] \zeta\left(\left\{g\left(s,\left(z_{n}\right)_{s}, H_{z_{n}}(s)\right)\right\}\right) d s \\
& \leq 2 \int_{0}^{t}\left[(t-s)^{r-1}-\left(t_{1}-s\right)^{r-1}\right] k_{1}(s) \zeta\left(\left\{\left(z_{n}\right)_{s}\right\}\right) d s \\
& +4\|l\|_{L^{1}} \int_{0}^{t}\left[(t-s)^{r-1}-\left(t_{1}-s\right)^{r-1}\right] k_{2}(s) \zeta\left(\left\{\left(z_{n}\right)_{s}\right\}\right) d s \\
& \leq 2\left(\int_{0}^{t}\left[(t-s)^{r-1}-\left(t_{1}-s\right)^{r-1}\right]^{\frac{1}{1-r_{1}}} d s\right)^{1-r_{1}}\left\|k_{1}\right\|_{L^{\frac{1}{r_{1}}}} c \zeta\left(\left\{z_{n}\right\}\right) \\
& +4\|l\|_{L^{1}}\left(\int_{0}^{t}\left[(t-s)^{r-1}-\left(t_{1}-s\right)^{r-1}\right]^{\frac{1}{1-r_{2}}} d s\right)^{1-r_{2}}\left\|k_{2}\right\|_{L^{\frac{1}{r_{2}}}} c \zeta\left(\left\{z_{n}\right\}\right) \\
& \leq 2\left(\int_{0}^{t}\left[(t-s)^{\vartheta_{1}}-\left(t_{1}-s\right)^{\vartheta_{1}}\right] d s\right)^{1-r_{1}}\left\|k_{1}\right\|_{L^{\frac{1}{r_{1}}}} c \zeta\left(\left\{z_{n}\right\}\right) \\
& +4\|l\|_{L^{1}}\left(\int_{0}^{t}\left[(t-s)^{\vartheta_{2}}-\left(t_{1}-s\right)^{\vartheta_{2}}\right] d s\right)^{1-r_{2}}\left\|k_{2}\right\|_{L^{\frac{1}{r_{2}}}} c \zeta\left(\left\{z_{n}\right\}\right) \\
& \leq \frac{2\left\|k_{1}\right\|_{L^{\frac{1}{r_{1}}}}}{\left(1+\vartheta_{1}\right)^{1-r_{1}}}\left[t^{1+\vartheta_{1}}-t_{1}^{1+\vartheta_{1}}+\left(t_{1}-t\right)^{1+\vartheta_{1}}\right]^{1-r_{1}} c \zeta\left(\left\{z_{n}\right\}\right) \\
& +\frac{4\left\|k_{2}\right\|_{L^{\frac{1}{r_{2}}}}\|l\|_{L^{1}}}{\left(1+\vartheta_{2}\right)^{1-r_{2}}}\left[t^{1+\vartheta_{2}}-t_{1}^{1+\vartheta_{2}}+\left(t_{1}-t\right)^{1+\vartheta_{2}}\right]^{1-r_{2}} c \zeta\left(\left\{z_{n}\right\}\right) \\
& \leq\left(2 \theta_{1}^{1-r_{1}}\left\|k_{1}\right\|_{L^{\frac{1}{r_{1}}}}\left(t_{1}-t\right)^{\left(1+\vartheta_{1}\right)\left(1-r_{1}\right)}+4 \theta_{2}^{1-r_{2}}\left\|k_{2}\right\|_{L^{\frac{1}{r_{2}}}}\|l\|_{L^{1}}\left(t_{1}-t\right)^{\left(1+\vartheta_{2}\right)\left(1-r_{2}\right)}\right) c \zeta\left(\left\{z_{n}\right\}\right) \\
& \leq\left(2 \theta_{1}^{1-r_{1}}\left\|k_{1}\right\|_{L^{\frac{1}{r_{1}}}}\left(t_{1}-t\right)^{r-r_{1}}+4 \theta_{2}^{1-r_{2}}\left\|k_{2}\right\|_{L^{\frac{1}{r_{2}}}}\|l\|_{L^{1}}\left(t_{1}-t\right)^{r-r_{2}}\right) c \zeta\left(\left\{z_{n}\right\}\right) \\
& \leq\left(2 c \theta_{1}^{1-r_{1}}\left\|k_{1}\right\|_{L^{\frac{1}{r_{1}}}}+4 c \theta_{2}^{1-r_{2}}\left\|k_{2}\right\|_{L^{\frac{1}{r_{2}}}}\|l\|_{L^{1}}\right)\left(t_{1}-t\right)^{r} \zeta\left(\left\{z_{n}\right\}\right) \\
& =K_{1}\left(t_{1}-t\right)^{r} \zeta(D) \text {. }
\end{aligned}
$$

In the same way, one can obtain 


$$
\begin{aligned}
& \zeta\left(\left(\mathcal{K}_{2} D\right)(t)\right)=\zeta\left(\left\{\left(\mathcal{K}_{2} z_{n}\right)(t)\right\}\right) \\
& =\zeta\left(\left\{\int_{t}^{t_{1}}\left(t_{1}-s\right)^{r-1} g\left(s,\left(z_{n}\right)_{s}, H_{z_{n}}(s)\right) d s\right\}\right) \\
& \leq 2 \int_{t}^{t_{1}}\left(t_{1}-s\right)^{r-1} \zeta\left(\left\{g\left(s,\left(z_{n}\right)_{s}, H_{z_{n}}(s)\right)\right\}\right) d s \\
& \leq 2 \int_{t}^{t_{1}}\left(t_{1}-s\right)^{r-1} k_{1}(s) \zeta\left(\left\{\left(z_{n}\right)_{s}\right\}\right) d s \\
& +4\|l\|_{L^{1}} \int_{t}^{t_{1}}\left(t_{1}-s\right)^{r-1} k_{2}(s) \zeta\left(\left\{\left(z_{n}\right)_{s}\right\}\right) d s \\
& \leq 2\left(\int_{t}^{t_{1}}\left(t_{1}-s\right)^{\frac{r-1}{1-r_{1}}} d s\right)^{1-r_{1}}\left\|k_{1}\right\|_{L^{\frac{1}{r_{1}}}} c \zeta\left(\left\{z_{n}\right\}\right) \\
& +4\|l\|_{L^{1}}\left(\int_{t}^{t_{1}}\left(t_{1}-s\right)^{\frac{r-1}{1-r_{2}}} d s\right)^{1-r_{2}}\left\|k_{2}\right\|_{L^{\frac{1}{r_{2}}}} c \zeta\left(\left\{z_{n}\right\}\right) \\
& \leq\left(2 \theta_{1}\left(t_{1}-t\right)^{\frac{1}{\theta_{1}}}\left\|k_{1}\right\|_{L^{\frac{1}{r_{1}}}}+4 \theta_{2}\left(t_{1}-t\right)^{\frac{1}{\theta_{2}}}\left\|k_{2}\right\|_{L^{\frac{1}{r_{2}}}}\|l\|_{L^{1}}\right) c \zeta\left(\left\{z_{n}\right\}\right) \\
& \leq\left(2 c \theta_{1}\left\|k_{1}\right\|_{L^{\frac{1}{r_{1}}}}+4 c \theta_{2}\left\|k_{2}\right\|_{L^{\frac{1}{r_{2}}}}\|l\|_{L^{1}}\right)\left(t_{1}-t\right)^{r} \zeta\left(\left\{z_{n}\right\}\right) \\
& =K_{2}\left(t_{1}-t\right)^{r} \zeta(D) \text {. }
\end{aligned}
$$

The conclusion follows.

Theorem 1. If assumptions (H1)-(H4) hold, then the dynamical system (1) is controllable on $V$.

Proof. We let constant

$$
K=\sup \left\{\left\|g\left(t, z_{t}, H_{z}(t)\right)\right\|:\left\|z_{t}\right\|_{L[-c, 0]} \leq c\left(\|\psi\|_{c}+R_{0}\right),\left\|H_{z}(t)\right\| \leq K_{0}, t \in V\right\},
$$

where $R_{0}=N_{0}\|\psi(0)\|+\frac{N_{0} r+2 N_{1}}{r^{2}}$, and

$$
K_{0}=\sup \left\{\left\|\int_{0}^{t} h\left(t, s, z_{s}\right) d s\right\|:(t, s) \in \Delta,\left\|z_{s}\right\|_{L[-c, 0]} \leq c\left(\|\psi\|_{c}+R_{0}\right)\right\} .
$$

From (H3), for an arbitrary function $z(\cdot) \in C(V ; E)$ and any $z_{1} \in E$, define a feedback control

$$
x_{z}(t):=\Lambda^{-1}\left(T_{0}\right)\left(z_{1}-\mathcal{N}\left(T_{0}\right)\left(\psi(0)+\mathcal{G}_{z}\left(T_{0}\right)\right)-\int_{0}^{T_{0}} \dot{\mathcal{N}}\left(T_{0}-s\right)\left(\mathcal{G}_{z}(s)-\mathcal{G}_{z}\left(T_{0}\right)\right) d s\right)(t), \quad t \in V,
$$

where

$$
T_{0}=\min \left\{T,\left(\frac{1}{K+Q_{1} N_{3}}\right)^{\frac{1}{r}},\left(\frac{r \Gamma^{3}(r+1)}{1+Q+2 l_{0} Q_{1} Q_{2} Q T^{r}\left(N_{0}+8 N_{1} T^{r}\right)}\right)^{\frac{1}{r}}\right\},
$$

and $N_{3}=Q_{2}\left(\left\|z_{1}\right\|+N_{0}\left(\|\psi(0)\|+\frac{K T^{r}}{r}\right)+\frac{2 K N_{1} T^{r}}{r^{2}}\right)$. Take $W=\left[-c, T_{0}\right]$. By considering Lemmas 1 and 7 , in what follows, it suffices to show that, when using this control, the operator $\mathcal{P}: C(W ; E) \rightarrow C(W ; E)$ defined by

$$
(\mathcal{P} z)(t)=\left\{\begin{array}{l}
\mathcal{N}(t)\left(\psi(0)+\mathcal{G}_{z}(t)+\mathcal{B}_{x_{z}}(t)\right) \\
+\int_{0}^{t} \dot{\mathcal{N}}(t-s)\left(\mathcal{G}_{z}(s)-\mathcal{G}_{z}(t)\right) d s+\int_{0}^{t} \dot{\mathcal{N}}(t-s)\left(\mathcal{B}_{x_{z}}(s)-\mathcal{B}_{x_{z}}(t)\right) d s, t \in\left[0, T_{0}\right], \\
\psi(t), t \in[-c, 0]
\end{array}\right.
$$

has a fixed point, from which it follows that this fixed point is a mild solution to the system (1) on $W$. Clearly, $(\mathcal{P} z)\left(T_{0}\right)=z_{1}$, which means that the control $x_{z}$ steers the system (1) from the initial function $\psi$ to $z_{1}$ in finite time $T_{0}$. Denote

$$
\Omega=\left\{z \in C(W ; E) \mid \sup _{t \in\left[0, T_{0}\right]}\left\|z_{t}\right\|_{L[-c, 0]} \leq c\left(\|\psi\|_{\mathcal{c}}+R_{0}\right) ; z(t)=\psi(t), t \in[-c, 0]\right\},
$$


then $\Omega$ is obviously a closed convex set in $C(W ; E)$. Subsequently, we will use Mönch fixed point theorem. To this end, we proceed the following four steps.

Step I. $\mathcal{P}(\Omega) \subseteq \Omega$. From (H3), we have

$$
\begin{aligned}
\left\|x_{z}(t)\right\| & \leq Q_{2}\left(\left\|z_{1}\right\|+N_{0}\|\psi(0)\|+N_{0} \frac{K T^{r}}{r}+\int_{0}^{T_{0}} \frac{N_{1}}{T_{0}-s}\left\|\mathcal{G}_{z}\left(T_{0}\right)-\mathcal{G}_{z}(s)\right\| d s\right) \\
& \leq Q_{2}\left(\left\|z_{1}\right\|+N_{0}\|\psi(0)\|+N_{0} \frac{K T^{r}}{r}+\frac{2 N_{1} K}{r} \int_{0}^{T_{0}} \frac{1}{T_{0}-s}\left(T_{0}-s\right)^{r} d s\right) \\
& \leq Q_{2}\left(\left\|z_{1}\right\|+N_{0}\left(\|\psi(0)\|+\frac{K T^{r}}{r}\right)+\frac{2 K N_{1} T^{r}}{r^{2}}\right)=N_{3}, \quad t \in V .
\end{aligned}
$$

From (H3) and (6), for any $z \in \Omega$ and $t \in\left[0, T_{0}\right]$, it follows that

$$
\begin{aligned}
\|(\mathcal{P} z)(t)\| \leq & N_{0}\left(\|\psi(0)\|+\frac{K T_{0}^{r}}{r}+\frac{Q_{1} N_{3} T_{0}^{r}}{r}\right) \\
& +\int_{0}^{t} \frac{N_{1}}{t-s} \frac{2 K(t-s)^{r}}{r} d s+\int_{0}^{t} \frac{N_{1}}{t-s} \frac{2 Q_{1} N_{3}}{r}(t-s)^{r} d s \\
\leq & N_{0}\left(\|\psi(0)\|+\frac{K T_{0}^{r}}{r}+\frac{Q_{1} N_{3} T_{0}^{r}}{r}\right)+\frac{2 N_{1} K T_{0}^{r}}{r^{2}}+\frac{2 Q_{1} N_{1} N_{3} T_{0}^{r}}{r^{2}} \\
\leq & N_{0}\|\psi(0)\|+\frac{\left(N_{0} r+2 N_{1}\right)\left(K+Q_{1} N_{3}\right)}{r^{2}} T_{0}^{r} \\
\leq & N_{0}\|\psi(0)\|+\frac{N_{0} r+2 N_{1}}{r^{2}}=R_{0} .
\end{aligned}
$$

On the other hand,

$$
\left\|(\mathcal{P} z)_{t}\right\|_{L[-c, 0]}=\int_{-c}^{0}(\mathcal{P} z)_{t}(\theta) d \theta= \begin{cases}\int_{t-c}^{0} \psi(s) d s+\int_{0}^{t}(\mathcal{P} z)(s) d s, & t \leq c, \\ \int_{t-c}^{t}(\mathcal{P} z)(s) d s, & t \geq c,\end{cases}
$$

which implies $\left\|(\mathcal{P} z)_{t}\right\|_{L[-c, 0]} \leq c\|\psi\|_{c}+c\|\mathcal{P} z\|_{C([0, t] ; E)}$. Then, one can obtain

$$
\sup _{t \in\left[0, T_{0}\right]}\left\|(\mathcal{P} z)_{t}\right\|_{L[-c, 0]} \leq c\left(\|\psi\|_{c}+R_{0}\right)
$$

It is clear that $(\mathcal{P} z)(t)=\psi(t)$ for any $z \in \Omega, t \in[-c, 0]$. Then, we conclude that $\mathcal{P}(\Omega) \subseteq \Omega$.

Step II. $\mathcal{P}: \Omega \rightarrow \Omega$ is equicontinuous.

For any $z \in \Omega$ and $t_{1}, t_{2} \in W=\left[-c, T_{0}\right]$ with $t_{1}<t_{2}$, we have the following discussion. (i) $0 \leq t_{1}<t_{2} \leq T_{0}$. Note that

$$
\begin{aligned}
& (\mathcal{P} z)\left(t_{2}\right)-(\mathcal{P} z)\left(t_{1}\right) \\
= & \left(\mathcal{N}\left(t_{2}\right)-\mathcal{N}\left(t_{1}\right)\right) \psi(0) \\
& +\mathcal{N}\left(t_{2}\right) \mathcal{G}_{z}\left(t_{2}\right)-\mathcal{N}\left(t_{1}\right) \mathcal{G}_{z}\left(t_{1}\right)+\mathcal{N}\left(t_{2}\right) \mathcal{B}_{x_{z}}\left(t_{2}\right)-\mathcal{N}\left(t_{1}\right) \mathcal{B}_{x_{z}}\left(t_{1}\right) \\
& +\int_{0}^{t_{2}} \dot{\mathcal{N}}\left(t_{2}-s\right)\left(\mathcal{G}_{z}(s)-\mathcal{G}_{z}\left(t_{2}\right)\right) d s-\int_{0}^{t_{1}} \dot{\mathcal{N}}\left(t_{1}-s\right)\left(\mathcal{G}_{z}(s)-\mathcal{G}_{z}\left(t_{1}\right)\right) d s \\
& +\int_{0}^{t_{2}} \dot{\mathcal{N}}\left(t_{2}-s\right)\left(\mathcal{B}_{x_{z}}(s)-\mathcal{B}_{x_{z}}\left(t_{2}\right)\right) d s-\int_{0}^{t_{1}} \dot{\mathcal{N}}\left(t_{1}-s\right)\left(\mathcal{B}_{x_{z}}(s)-\mathcal{B}_{x_{z}}\left(t_{1}\right)\right) d s
\end{aligned}
$$

Clearly,

$$
\left\|(\mathcal{P} z)\left(t_{2}\right)-(\mathcal{P} z)\left(t_{1}\right)\right\| \leq\left\|\mathfrak{O}_{1}\right\|+\left\|\mathfrak{O}_{2}\right\|+\left\|\mathfrak{O}_{3}\right\|+\left\|\mathfrak{O}_{4}\right\|,
$$

where

$\mathfrak{O}_{1}=\left(\mathcal{N}\left(t_{2}\right)-\mathcal{N}\left(t_{1}\right)\right) \psi(0)$,

$$
\begin{aligned}
& \mathfrak{O}_{2}=\left(\mathcal{N}\left(t_{2}\right) \mathcal{G}_{z}\left(t_{2}\right)-\mathcal{N}\left(t_{1}\right) \mathcal{G}_{z}\left(t_{1}\right)\right)+\left(\mathcal{N}\left(t_{2}\right) \mathcal{B}_{x_{z}}\left(t_{2}\right)-\mathcal{N}\left(t_{1}\right) \mathcal{B}_{x_{z}}\left(t_{1}\right)\right) \\
& \mathfrak{O}_{3}=\int_{0}^{t_{2}} \dot{\mathcal{N}}\left(t_{2}-s\right)\left(\mathcal{G}_{z}(s)-\mathcal{G}_{z}\left(t_{2}\right)\right) d s-\int_{0}^{t_{1}} \dot{\mathcal{N}}\left(t_{1}-s\right)\left(\mathcal{G}_{z}(s)-\mathcal{G}_{z}\left(t_{1}\right)\right) d s \\
& \mathfrak{O}_{4}=\int_{0}^{t_{2}} \dot{\mathcal{N}}\left(t_{2}-s\right)\left(\mathcal{B}_{x_{z}}(s)-\mathcal{B}_{x_{z}}\left(t_{2}\right)\right) d s-\int_{0}^{t_{1}} \dot{\mathcal{N}}\left(t_{1}-s\right)\left(\mathcal{B}_{x_{z}}(s)-\mathcal{B}_{x_{z}}\left(t_{1}\right)\right) d s
\end{aligned}
$$


The strong continuity of $\mathcal{N}(\cdot)$ indicates that $\left\|\mathfrak{O}_{1}\right\| \rightarrow 0$, as $\left|t_{1}-t_{2}\right| \rightarrow 0$. By Lemma 7 , one can obtain

$$
\begin{aligned}
\left\|\mathcal{O}_{2}\right\| \leq & \left\|\mathcal{N}\left(t_{2}\right)\right\|_{\mathcal{L}(E)}\left\|\mathcal{G}_{z}\left(t_{2}\right)-\mathcal{G}_{z}\left(t_{1}\right)\right\|+\left\|\left(\mathcal{N}\left(t_{2}\right)-\mathcal{N}\left(t_{1}\right)\right) \mathcal{G}_{z}\left(t_{1}\right)\right\| \\
& +\left\|\mathcal{N}\left(t_{2}\right)\right\|_{\mathcal{L}(E)}\left\|\mathcal{B}_{x_{z}}\left(t_{2}\right)-\mathcal{B}_{x_{z}}\left(t_{1}\right)\right\|+\left\|\left(\mathcal{N}\left(t_{2}\right)-\mathcal{N}\left(t_{1}\right)\right) \mathcal{B}_{x_{z}}\left(t_{1}\right)\right\| \\
\leq \quad & N_{0}\left[\left|\mathcal{G}_{z}\right|\right]_{C^{r}}\left(t_{2}-t_{1}\right)^{r}+\int_{t_{1}}^{t_{2}}\left\|\mathcal{N}(s) \mathcal{G}_{z}\left(t_{1}\right)\right\| d s \\
& +N_{0} Q_{1} N_{3} \frac{t_{2}{ }^{r}-t_{1}{ }^{r}+\left(t_{2}-t_{1}\right)^{r}}{r}+N_{0} \int_{t_{1}}^{t_{2}} \frac{\left\|\mathcal{B}_{z}(s)\right\|}{\left(t_{2}-s\right)^{1-r}} d s \\
& +\left\|\left(\mathcal{N}\left(t_{2}\right)-\mathcal{N}\left(t_{1}\right)\right)\right\|_{\mathcal{L}(E)} \frac{Q_{1} N_{3} T_{0}^{r}}{r} \\
\leq \quad & N_{0}\left[\left|\mathcal{G}_{z}\right|\right]_{C^{r}}\left(t_{2}-t_{1}\right)^{r}+N_{1}\left[\left|\mathcal{G}_{z}\right|\right]_{C^{r}} \int_{t_{1}}^{t_{2}} \frac{s^{r}}{s} d s \\
& +N_{0} Q_{1} N_{3} \frac{t_{2}{ }^{r}-t_{1}{ }^{r}+\left(t_{2}-t_{1}\right)^{r}}{r}+N_{0} Q_{1} N_{3} \frac{\left(t_{2}-t_{1}\right)^{r}}{r} \\
& +\left\|\left(\mathcal{N}\left(t_{2}\right)-\mathcal{N}\left(t_{1}\right)\right)\right\|_{\mathcal{L}(E)} \frac{Q_{1} N_{3} T_{0}^{r}}{r} \\
\leq & N_{0} \frac{2 K}{\Gamma(r+1)}\left(t_{2}-t_{1}\right)^{r}+N_{1} \frac{2 K}{\Gamma(r+1)} \frac{\left(t_{2}-t_{1}\right)^{r}}{r}+N_{0} Q_{1} N_{3} \frac{t_{2}{ }^{r}-t_{1}{ }^{r}+\left(t_{2}-t_{1}\right)^{r}}{r} \\
& +N_{0} Q_{1} N_{3} \frac{\left(t_{2}-t_{1}\right)^{r}}{r}+\left\|\left(\mathcal{N}\left(t_{2}\right)-\mathcal{N}\left(t_{1}\right)\right)\right\| \|_{\mathcal{L}(E)} \frac{Q_{1} N_{3} T_{0}^{r}}{r} \rightarrow 0, a s\left|t_{1}-t_{2}\right| \rightarrow 0 .
\end{aligned}
$$

By means of Lemma 7, we can obtain

$$
\begin{aligned}
\left\|\mathfrak{O}_{3}\right\| \leq & \int_{0}^{t_{1}}\left\|\dot{\mathcal{N}}\left(\left(t_{2}-t_{1}\right)+s\right)-\dot{\mathcal{N}}(s)\right\|_{\mathcal{L}(E)}\left\|\mathcal{G}_{z}\left(t_{1}-s\right)-\mathcal{G}_{z}\left(t_{1}\right)\right\| d s \\
& +\left\|\int_{0}^{t_{1}} \dot{\mathcal{N}}\left(\left(t_{2}-t_{1}\right)+s\right)\left(\mathcal{G}_{z}\left(t_{1}\right)-\mathcal{G}_{z}\left(t_{2}\right)\right) d s\right\| \\
& +\int_{t_{1}}^{t_{2}}\left\|\dot{\mathcal{N}}\left(t_{2}-s\right)\right\|\left\|_{\mathcal{L}(E)}\right\| \mathcal{G}_{z}(s)-\mathcal{G}_{z}\left(t_{2}\right) \| d s \\
\leq & \int_{0}^{t_{1}} \int_{s}^{s+\left(t_{2}-t_{1}\right)}\|\ddot{\mathcal{N}}(\xi)\|_{\mathcal{L}(E)}\left[\left|\mathcal{G}_{z}\right|\right]_{C^{r}} s^{r} d \xi d s \\
& +\left\|\mathcal{N}\left(t_{2}\right)-\mathcal{N}\left(\left(t_{2}-t_{1}\right)\right)\right\|_{\mathcal{L}(E)}\left\|\mathcal{G}_{z}\left(t_{1}\right)-\mathcal{G}_{z}\left(t_{2}\right)\right\|+N_{1}\left[\left|\mathcal{G}_{z}\right|\right]_{C^{r}} \int_{t_{1}}^{t_{2}}\left(t_{2}-s\right)^{r-1} d s \\
\leq & {\left[\left|\mathcal{G}_{z}\right|\right]_{C^{r}} N_{2} \int_{0}^{t_{1}} \int_{s}^{s+\left(t_{2}-t_{1}\right)} \xi^{r-2} d \xi d s+2 N_{0}\left[\left|\mathcal{G}_{z}\right|\right]_{C^{r}}\left(t_{2}-t_{1}\right)^{r}+N_{1}\left[\left|\mathcal{G}_{z}\right|\right]_{C^{r}} \frac{\left(t_{2}-t_{1}\right)^{r}}{r} } \\
\leq & \frac{2\left(t_{2}-t_{1}\right)^{r}}{r(1-r)}\left[\left|\mathcal{G}_{z}\right|\right]_{C^{r}} N_{2}+2 N_{0}\left[\left|\mathcal{G}_{z}\right|\right]_{C^{r}}\left(t_{2}-t_{1}\right)^{r}+\frac{N_{1}\left[\left|\mathcal{G}_{z}\right|\right]_{C^{r}}\left(t_{2}-t_{1}\right)^{r}}{r} \\
\leq & \frac{4 N_{2} K\left(t_{2}-t_{1}\right)^{r}}{r(1-r) \Gamma(r+1)}+\frac{4 N_{0} K\left(t_{2}-t_{1}\right)^{r}}{\Gamma(r+1)}+\frac{2 N_{1} K\left(t_{2}-t_{1}\right)^{r}}{r \Gamma(r+1)} \rightarrow 0, a s\left|t_{1}-t_{2}\right| \rightarrow 0 .
\end{aligned}
$$

Similarly,

$$
\begin{aligned}
\left\|\mathcal{O}_{4}\right\| \leq & \frac{2\left(t_{2}-t_{1}\right)^{r}}{r(1-r)}\left[\left|\mathcal{B}_{x_{z}}\right|\right]_{C^{r}} N_{2}+2 N_{0}\left[\left|\mathcal{B}_{x_{z}}\right|\right]_{C^{r}}\left(t_{2}-t_{1}\right)^{r}+N_{1}\left[\left|\mathcal{B}_{x_{z}}\right|\right]_{C^{r}} \frac{\left(t_{2}-t_{1}\right)^{r}}{r} \\
\leq & \frac{4 Q_{1} N_{2} N_{3}\left(t_{2}-t_{1}\right)^{r}}{r(1-r) \Gamma(r+1)}+\frac{4 Q_{1} N_{0} N_{3}\left(t_{2}-t_{1}\right)^{r}}{\Gamma(r+1)}+\frac{2 Q_{1} N_{1} N_{3}\left(t_{2}-t_{1}\right)^{r}}{r \Gamma(r+1)} \\
& \rightarrow 0, \text { as }\left|t_{1}-t_{2}\right| \rightarrow 0 .
\end{aligned}
$$

(ii) $-c \leq t_{1}<t_{2} \leq 0$. From the continuity of $\psi(\cdot)$, we have

$$
\left\|(\mathcal{P} z)\left(t_{2}\right)-(\mathcal{P} z)\left(t_{1}\right)\right\|=\left\|\psi\left(t_{2}\right)-\psi\left(t_{1}\right)\right\| \rightarrow 0, \text { as }\left|t_{1}-t_{2}\right| \rightarrow 0 .
$$

(iii) $-c \leq t_{1}<0<t_{2} \leq T_{0}$. Then, 


$$
\begin{aligned}
\left\|(\mathcal{P} z)\left(t_{2}\right)-(\mathcal{P} z)\left(t_{1}\right)\right\| \leq & \left\|(\mathcal{P} z)\left(t_{2}\right)-(\mathcal{P} z)(0)\right\|+\left\|(\mathcal{P} z)(0)-(\mathcal{P} z)\left(t_{1}\right)\right\| \\
\leq & \left\|\left(\mathcal{N}\left(t_{2}\right)-I\right) \psi(0)\right\|+\left\|\mathcal{N}\left(t_{2}\right) \mathcal{G}_{z}\left(t_{2}\right)\right\|+\left\|\mathcal{N}\left(t_{2}\right) \mathcal{B}_{x_{z}}\left(t_{2}\right)\right\| \\
& +\left\|\int_{0}^{t_{2}} \dot{\mathcal{N}}\left(t_{2}-s\right)\left(\mathcal{G}_{z}(s)-\mathcal{G}_{z}\left(t_{2}\right)\right) d s\right\| \\
& +\left\|\int_{0}^{t_{2}} \dot{\mathcal{N}}\left(t_{2}-s\right)\left(\mathcal{B}_{x_{z}}(s)-\mathcal{B}_{x_{z}}\left(t_{2}\right)\right) d s\right\|+\left\|\psi(0)-\psi\left(t_{1}\right)\right\| \\
\leq \quad & \left\|\left(\mathcal{N}\left(t_{2}\right)-I\right) \psi(0)\right\|+\frac{N_{0}\left(K+Q_{1} N_{3}\right)}{r} t_{2}^{r} \\
& +\frac{2 N_{1}\left(K+Q_{1} N_{3}\right)}{r} \int_{0}^{t_{2}} \frac{1}{t_{2}-s}\left(t_{2}-s\right)^{r} d s+\left\|\psi(0)-\psi\left(t_{1}\right)\right\| \\
\leq & \left\|\left(\mathcal{N}\left(t_{2}\right)-I\right) \psi(0)\right\|+\frac{N_{0}\left(K+Q_{1} N_{3}\right)}{r} t_{2}^{r} \\
& +\frac{2 N_{1}\left(K+Q_{1} N_{3}\right)}{r^{2}} t_{2}^{r}+\left\|\psi(0)-\psi\left(t_{1}\right)\right\| \rightarrow 0, \text { as }\left|t_{1}-t_{2}\right| \rightarrow 0 .
\end{aligned}
$$

Thus, $\left\|(\mathcal{P} z)\left(t_{2}\right)-(\mathcal{P} z)\left(t_{1}\right)\right\| \rightarrow 0$, as $\left|t_{1}-t_{2}\right| \rightarrow 0$, for every $z \in \Omega$. This deduces that $\mathcal{P}: \Omega \rightarrow \Omega$ is equicontinuous.

Step III. $\mathcal{P}$ is continuous on $\Omega$.

Let $y_{n}$ be a sequence, such that $y_{n} \rightarrow y$ in $\Omega$ as $n \rightarrow \infty$. We only consider the case $t \in\left[0, T_{0}\right]$ since the continuity of operator $\mathcal{P}$ is obvious under the case $t \in[-c, 0]$.

For each $t \in\left[0, T_{0}\right]$, one has

$$
\begin{aligned}
\left\|\left(\mathcal{P} y_{n}\right)(t)-(\mathcal{P} y)(t)\right\| \leq & N_{0}\left\|\mathcal{G}_{y_{n}}(t)-\mathcal{G}_{y}(t)\right\|+N_{0}\left\|\mathcal{B}_{x_{y_{n}}}(t)-\mathcal{B}_{x_{y}}(t)\right\| \\
& +\int_{0}^{t}\left\|\dot{\mathcal{N}}(t-s)\left[\left(\mathcal{G}_{y_{n}}(s)-\mathcal{G}_{y_{n}}(t)\right)-\left(\mathcal{G}_{y}(s)-\mathcal{G}_{y}(t)\right)\right]\right\| d s \\
& +\int_{0}^{t}\left\|\dot{\mathcal{N}}(t-s)\left[\left(\mathcal{B}_{x_{y_{n}}}(s)-\mathcal{B}_{x_{y_{n}}}(t)\right)-\left(\mathcal{B}_{x_{y}}(s)-\mathcal{B}_{x_{y}}(t)\right)\right]\right\| d s \\
\leq & N_{0} \int_{0}^{t}(t-s)^{r-1}\left\|g\left(s,\left(y_{n}\right)_{s}, H_{y_{n}}(s)\right)-g\left(s, y_{s}, H_{y}(s)\right)\right\| d s \\
& +\frac{N_{0} Q_{1}}{\Gamma(r)} \int_{0}^{t}(t-s)^{r-1}\left\|x_{y_{n}}(s)-x_{y}(s)\right\| d s \\
& +\int_{0}^{t}\left\|\dot{\mathcal{N}}(t-s)\left[\left(\mathcal{G}_{y_{n}}(s)-\mathcal{G}_{y_{n}}(t)\right)-\left(\mathcal{G}_{y}(s)-\mathcal{G}_{y}(t)\right)\right]\right\| d s \\
& +\int_{0}^{t}\left\|\dot{\mathcal{N}}(t-s)\left[\left(\mathcal{B}_{x_{y_{n}}}(s)-\mathcal{B}_{x_{y_{n}}}(t)\right)-\left(\mathcal{B}_{x_{y}}(s)-\mathcal{B}_{x_{y}}(t)\right)\right]\right\| d s
\end{aligned}
$$

It is easy to check that the following inequalities hold:

$$
\begin{aligned}
(\cdot-s)^{r-1}\left\|g\left(s,\left(y_{n}\right)_{s}, H_{y_{n}}(s)\right)-g\left(s, y_{s}, H_{y}(s)\right)\right\| & \leq 2 K(\cdot-s)^{r-1} \in L^{1}\left(\left[0, T_{0}\right], R^{+}\right), \\
(\cdot-s)^{r-1}\left\|x_{y_{n}}(s)-x_{y}(s)\right\| \leq 2 N_{3}(\cdot-s)^{r-1} \in L^{1}\left(\left[0, T_{0}\right], R^{+}\right), & \leq \frac{N_{1}}{(\cdot-s)} \frac{2 K(\cdot-s)^{r}}{r} \\
\left\|\dot{N}(\cdot-s)\left[\left(\mathcal{G}_{y_{n}}(s)-\mathcal{G}_{y_{n}}(\cdot)\right)-\left(\mathcal{G}_{y}(s)-\mathcal{G}_{y}(\cdot)\right)\right]\right\| & \leq \frac{2 N_{1} K}{r}(\cdot-s)^{r-1} \in L^{1}\left(\left[0, T_{0}\right], R^{+}\right), \\
\left\|\dot{\mathcal{N}}(\cdot-s)\left[\left(\mathcal{B}_{x_{y_{n}}}(s)-\mathcal{B}_{x_{y_{n}}}(\cdot)\right)-\left(\mathcal{B}_{x_{y}}(s)-\mathcal{B}_{x_{y}}(\cdot)\right)\right]\right\| & \leq \frac{N_{1}}{(\cdot-s)} \frac{2 Q_{1} N_{3}(\cdot-s)^{r}}{r} \\
& \leq \frac{2 Q_{1} N_{1} N_{3}}{r}(\cdot-s)^{r-1} \in L^{1}\left(\left[0, T_{0}\right], R^{+}\right) .
\end{aligned}
$$

Moreover, one has

$$
\begin{aligned}
& \left\|x_{y_{n}}(s)-x_{y}(s)\right\| \\
\leq \quad & Q_{2}\left(N_{0}\left\|\mathcal{G}_{y_{n}}\left(T_{0}\right)-\mathcal{G}_{y}\left(T_{0}\right)\right\|+\int_{0}^{T_{0}}\left\|\dot{\mathcal{N}}\left(T_{0}-s\right)\left[\left(\mathcal{G}_{y_{n}}(s)-\mathcal{G}_{y_{n}}(t)\right)-\left(\mathcal{G}_{y}(s)-\mathcal{G}_{y}(t)\right)\right]\right\| d s\right),
\end{aligned}
$$

and

$$
\begin{aligned}
\left\|\dot{\mathcal{N}}\left(T_{0}-s\right)\left[\left(\mathcal{G}_{y_{n}}(s)-\mathcal{G}_{y_{n}}(\cdot)\right)-\left(\mathcal{G}_{y}(s)-\mathcal{G}_{y}(\cdot)\right)\right]\right\| & \leq \frac{N_{1}}{\left(T_{0}-s\right)} \frac{2 K(\cdot-s)^{r}}{r} \\
& \leq \frac{2 N_{1} K}{r}(\cdot-s)^{r-1} \in L^{1}\left(\left[0, T_{0}\right], R^{+}\right) .
\end{aligned}
$$

Then, Lebesgue's domination convergence theorem implies that $\left\|\left(\mathcal{P} y_{n}\right)(t)-(\mathcal{P} y)(t)\right\| \rightarrow 0$, as $n \rightarrow+\infty$. From Ascoli-Arzelà theorem, it follows that $\left\|\mathcal{P} y_{n}-\mathcal{P} y\right\|_{C(W ; E)} \rightarrow 0$, as $n \rightarrow+\infty$. The proof is completed. 
Step IV. Mönch's condition holds.

Suppose $B=\overline{c o} \mathcal{P}(\Omega)$. From Step I and II, it is not difficult to check that $\mathcal{P}(B) \subseteq B$ and $B$ is equicontinuous.

For any $z \in B$, we take

$$
(\mathcal{P} z)(t)=\left\{\begin{array}{l}
\left(\mathcal{P}_{1} z\right)(t)+\left(\mathcal{P}_{2} z\right)(t)+\left(\mathcal{P}_{3} z\right)(t)+\left(\mathcal{P}_{4} z\right)(t), t \in\left[0, T_{0}\right], \\
\psi(t), t \in[-\mathcal{c}, 0],
\end{array}\right.
$$

where

$$
\begin{aligned}
& \left(\mathcal{P}_{1} z\right)(t)=\mathcal{N}(t) \psi(0)+\mathcal{N}(t) \mathcal{G}_{z}(t) \\
& \left(\mathcal{P}_{2} z\right)(t)=\int_{0}^{t} \dot{\mathcal{N}}(t-s)\left(\mathcal{G}_{z}(s)-\mathcal{G}_{z}(t)\right) d s \\
& \left(\mathcal{P}_{3} z\right)(t)=\mathcal{N}(t) \mathcal{B}_{x_{z}}(t) \\
& \left(\mathcal{P}_{4} z\right)(t)=\int_{0}^{t} \dot{\mathcal{N}}(t-s)\left(\mathcal{B}_{x_{z}}(s)-\mathcal{B}_{x_{z}}(t)\right) d s .
\end{aligned}
$$

Suppose bounded set $D_{0} \subset B$ is countable and $D_{0} \subset \overline{c o}\left(\left\{z_{0}\right\} \cup \mathcal{P}\left(D_{0}\right)\right)$, we shall show that $\zeta\left(D_{0}\right)=0$. Without loss of generality, we may suppose that $D_{0}=\left\{z_{n}\right\}_{n=1}^{\infty}$.

From Lemmas 4 (4) and 6 and Hypothesis (H4) (i), (ii), for any $s \in V$, we have

$$
\begin{aligned}
\zeta\left(\left\{g\left(s,\left(z_{n}\right)_{s}, H_{z_{n}}(s)\right)\right\}\right) & \leq k_{1}(s) \zeta\left(\left\{\left(z_{n}\right)_{s}\right\}\right)+k_{2}(s) \zeta\left(\left\{\int_{0}^{s} h\left(s, \eta,\left(z_{n}\right)_{\eta}\right) d \eta\right\}\right) \\
& \leq k_{1}(s) \zeta\left(\left\{\left(z_{n}\right)_{s}\right\}\right)+2 k_{2}(s) \int_{0}^{s} \zeta\left(\left\{h\left(s, \eta,\left(z_{n}\right)_{\eta}\right)\right\}\right) d \eta \\
& \leq k_{1}(s) \zeta\left(\left\{\left(z_{n}\right)_{s}\right\}\right)+2 k_{2}(s) \int_{0}^{s} l(\eta) \zeta\left(\left\{\left(z_{n}\right)_{\eta}\right\}\right) d \eta \\
& \leq\left(k_{1}(s)+2 k_{2}(s)\|l\|_{L^{1}}\right) c \zeta\left(\left\{z_{n}\right\}\right) .
\end{aligned}
$$

Then, for any $t \in\left[0, T_{0}\right]$, by Lemmas 3 and 6 , one has

$$
\begin{aligned}
& 2 \int_{0}^{t}(t-s)^{r-1} \zeta\left(\left\{g\left(s,\left(z_{n}\right)_{s}, H_{z_{n}}(s)\right)\right\}\right) d s \\
\leq & 2 \int_{0}^{t}(t-s)^{r-1} k_{1}(s) d s \cdot c \zeta\left(\left\{z_{n}\right\}\right)+4\|l\|_{L^{1}} \int_{0}^{t}(t-s)^{r-1} k_{2}(s) d s \cdot c \zeta\left(\left\{z_{n}\right\}\right) \\
\leq & 2\left(\int_{0}^{t}\left[(t-s)^{r-1}\right]^{\frac{1}{1-r_{1}}} d s\right)^{1-r_{1}}\left\|k_{1}\right\|_{L^{\frac{1}{r_{1}}}} \cdot c \zeta\left(\left\{z_{n}\right\}\right) \\
& +4\|l\|_{L^{1}}\left(\int_{0}^{t}\left[(t-s)^{r-1}\right]^{\frac{1}{1-r_{2}}} d s\right)^{1-r_{2}}\left\|k_{2}\right\|_{L^{\frac{1}{r_{2}}}} \cdot c \zeta\left(\left\{z_{n}\right\}\right) \\
\leq & \left(2\left(\frac{1-r_{1}}{r-r_{1}}\right)^{1-r_{1}} t^{r-r_{1}}\left\|k_{1}\right\|_{L^{\frac{1}{r_{1}}}}+4\|l\|_{L^{1}}\left(\frac{1-r_{2}}{r-r_{2}}\right)^{1-r_{2}} t^{r-r_{2}}\left\|k_{2}\right\|_{L^{\frac{1}{r_{2}}}}\right) c \zeta\left(\left\{z_{n}\right\}\right) \\
\leq & \left(2 c \theta_{1}^{1-r_{1}}\left\|k_{1}\right\|_{L^{\frac{1}{r_{1}}}}+4 c \theta_{2}^{1-r_{2}}\left\|k_{2}\right\|_{L^{\frac{1}{r_{2}}}}\|l\|_{L^{1}}\right) t^{r} \zeta\left(\left\{z_{n}\right\}\right) \\
\leq & K_{1} T_{0}^{r} \zeta\left(\left\{z_{n}\right\}\right) .
\end{aligned}
$$

Therefore,

$$
\begin{aligned}
\zeta\left(\left(\mathcal{P}_{1} D_{0}\right)(t)\right) & =\zeta\left(\left\{\mathcal{N}(t) \psi(0)+\mathcal{N}(t) \frac{1}{\Gamma(r)} \int_{0}^{t} \frac{g\left(s,\left(z_{n}\right)_{s}, H_{z_{n}}(s)\right)}{(t-s)^{1-r}} d s\right\}\right) \\
& \leq \frac{2 N_{0}}{\Gamma(r)} \int_{0}^{t}(t-s)^{r-1} \zeta\left(\left\{g\left(s,\left(z_{n}\right)_{s}, H_{z_{n}}(s)\right)\right\}\right) d s \\
& \leq \frac{N_{0} K_{1}}{\Gamma(r)} T_{0}^{r} \zeta\left(\left\{z_{n}\right\}\right) \\
& =\frac{N_{0} K_{1}}{\Gamma(r)} T_{0}^{r} \zeta\left(D_{0}\right), t \in\left[0, T_{0}\right] .
\end{aligned}
$$

For $t \in\left[0, T_{0}\right]$, in view of Lemmas 4 (4) and 8 , we get 


$$
\begin{aligned}
& \zeta\left(\left(\mathcal{P}_{2} D_{0}\right)(t)\right)=\zeta\left(\left\{\int_{0}^{t} \dot{\mathcal{N}}(t-s) \frac{1}{\Gamma(r)}\left(\int_{0}^{s} \frac{g\left(y,\left(z_{n}\right)_{y}, H_{z_{n}}(y)\right)}{(s-y)^{1-r}} d y-\int_{0}^{t} \frac{g\left(y,\left(z_{n}\right)_{y}, H_{z_{n}}(y)\right)}{(t-y)^{1-r}} d y\right) d s\right\}\right) \\
& \leq \frac{4 N_{1}}{\Gamma(r)} \int_{0}^{t} \frac{1}{t-s}\left(\int _ { 0 } ^ { s } ( ( s - y ) ^ { r - 1 } - ( t - y ) ^ { r - 1 } ) \zeta \left(\left\{g\left(y,\left(z_{n}\right)_{y}, H_{z_{n}}(y)\right) d y\right) d s\right.\right. \\
& +\frac{4 N_{1}}{\Gamma(r)} \int_{0}^{t} \frac{1}{t-s}\left(\int_{s}^{t}(t-y)^{r-1} \zeta\left(\left\{g\left(y,\left(z_{n}\right)_{y}, H_{z_{n}}(y)\right)\right\}\right) d y\right) d s \\
& \leq \frac{4 N_{1}}{\Gamma(r)} \int_{0}^{t} \frac{1}{t-s}\left[K_{1}(t-s)^{r} \zeta\left(\left\{z_{n}\right\}\right)\right] d s \\
& +\frac{4 N_{1}}{\Gamma(r)} \int_{0}^{t} \frac{1}{t-s}\left[K_{2}(t-s)^{r} \zeta\left(\left\{z_{n}\right\}\right)\right] d s \\
& \leq \frac{4 N_{1} K_{1}}{\Gamma(r+1)} t^{r} \zeta\left(\left\{z_{n}\right\}\right)+\frac{4 N_{1} K_{2}}{\Gamma(r+1)} t^{r} \zeta\left(\left\{z_{n}\right\}\right) \\
& \leq \frac{4 N_{1}\left(K_{1}+K_{2}\right)}{\Gamma(r+1)} t^{r} \zeta\left(\left\{z_{n}\right\}\right) \\
& \leq \frac{4 N_{1}\left(K_{1}+K_{2}\right)}{\Gamma(r+1)} T_{0}^{r} \zeta\left(D_{0}\right) \text {. } \\
& \zeta\left(\left\{\mathcal{B}_{x_{z_{n}}}(t)\right\}\right) \leq \frac{2 l_{0} Q_{1} Q_{2}}{\Gamma(r)} \int_{0}^{t}(t-s)^{r-1} \zeta\left(\left\{z_{1}-\mathcal{N}\left(T_{0}\right) \psi_{0}-\mathcal{N}\left(T_{0}\right) \mathcal{G}_{z_{n}}\left(T_{0}\right)\right\}\right) d s \\
& +\frac{2 l_{0} Q_{1} Q_{2}}{\Gamma(r)} \int_{0}^{t}(t-s)^{r-1} \zeta\left(\left\{\int_{0}^{T_{0}} \dot{\mathcal{N}}\left(T_{0}-s\right)\left(\mathcal{G}_{z_{n}}(s)-\mathcal{G}_{z_{n}}\left(T_{0}\right)\right) d s\right\}\right) d s \\
& \leq \frac{2 l_{0} Q_{1} Q_{2}}{\Gamma(r)} \int_{0}^{t}(t-s)^{r-1}\left[\zeta\left(\left\{\left(\mathcal{P}_{1} D_{0}\right)\left(T_{0}\right)\right\}\right)+\zeta\left(\left\{\left(\mathcal{P}_{2} D_{0}\right)\left(T_{0}\right)\right\}\right)\right] d s \\
& \leq \frac{2 l_{0} Q_{1} Q_{2} T^{r}}{\Gamma(r+1)}\left(\frac{N_{0} K_{1}}{\Gamma(r)}+\frac{4 N_{1}\left(K_{1}+K_{2}\right)}{\Gamma(r+1)}\right) T_{0}^{r} \zeta\left(D_{0}\right) \\
& \leq \frac{2 l_{0} Q_{1} Q_{2} T^{r}\left(N_{0} K_{1}+4 N_{1}\left(K_{1}+K_{2}\right)\right)}{\Gamma^{2}(r+1)} T_{0}^{r} \zeta\left(D_{0}\right) \\
& =\frac{2 l_{0} Q_{1} Q_{2} Q T^{r}}{\Gamma^{2}(r+1)} T_{0}^{r} \zeta\left(D_{0}\right), t \in\left[0, T_{0}\right],
\end{aligned}
$$

and this indicates

$$
\zeta\left(\left(\mathcal{P}_{3} D_{0}\right)(t)\right) \leq \frac{2 l_{0} N_{0} Q_{1} Q_{2} Q T^{r}}{\Gamma^{2}(r+1)} T_{0}^{r} \zeta\left(D_{0}\right), t \in\left[0, T_{0}\right] .
$$

By means of Lemma 4 (4), Hypothesis (H3) and (9), for $t \in\left[0, T_{0}\right]$, one derives

$$
\begin{aligned}
\zeta\left(\left(\mathcal{P}_{4} D_{0}\right)(t)\right)= & \zeta\left(\left\{\int_{0}^{t} \dot{\mathcal{N}}(t-s) \frac{1}{\Gamma(r)}\left(\int_{0}^{s} \frac{\mathcal{B} x_{z_{n}}(y)}{(s-y)^{1-r}} d y-\int_{0}^{t} \frac{\mathcal{B} x_{z_{n}}(y)}{(t-y)^{1-r}} d y\right) d s\right\}\right) \\
\leq & \frac{4 N_{1}}{\Gamma(r)} \int_{0}^{t} \frac{1}{t-s}\left(\int_{0}^{s}\left((s-y)^{r-1}-(t-y)^{r-1}\right) \zeta\left(\left\{\mathcal{B} x_{z_{n}}(y)\right\}\right) d y\right) d s \\
& +\frac{4 N_{1}}{\Gamma(r)} \int_{0}^{t} \frac{1}{t-s}\left(\int_{s}^{t}(t-y)^{r-1} \zeta\left(\left\{\mathcal{B} x_{z_{n}}(t)\right\}\right) d y\right) d s \\
\leq & \frac{4 N_{1} \zeta\left(\left\{\mathcal{B} x_{z_{n}}(t)\right\}\right)}{\Gamma(r)} \int_{0}^{t} \frac{1}{t-s}\left(\frac{s^{r}}{r}-\frac{t^{r}-(t-s)^{r}}{r}+\frac{(t-s)^{r}}{r}\right) d s \\
\leq & \frac{4 N_{1} \zeta\left(\left\{\mathcal{B} x_{z_{n}}(t)\right\}\right)}{\Gamma(r+1)} \int_{0}^{t} \frac{1}{t-s}\left(s^{r}-t^{r}+2(t-s)^{r}\right) d s \\
\leq & \frac{4 N_{1} \zeta\left(\left\{\mathcal{B} x_{z_{n}}(t)\right\}\right)}{\Gamma(r+1)} \int_{0}^{t} \frac{1}{t-s} \cdot 2(t-s)^{r} d s \\
\leq & \frac{8 N_{1} \zeta\left(\left\{\mathcal{B} x_{z_{n}}(t)\right\}\right)}{\Gamma(r+1)} \int_{0}^{t}(t-s)^{r-1} d s \\
\leq & \frac{16 l_{0} Q_{1} Q_{2} Q N_{1} T^{2 r}}{r \Gamma^{3}(r+1)} T_{0}^{r} \zeta\left(D_{0}\right) .
\end{aligned}
$$


Thus, (7), (8), (10) and (11) imply that

$$
\begin{aligned}
\zeta\left(\left(\mathcal{P} D_{0}\right)(t)\right) \leq & \zeta\left(\left(\mathcal{P}_{1} D_{0}\right)(t)\right)+\zeta\left(\left(\mathcal{P}_{2} D_{0}\right)(t)\right)+\zeta\left(\left(\mathcal{P}_{3} D_{0}\right)(t)\right)+\zeta\left(\left(\mathcal{P}_{4} D_{0}\right)(t)\right) \\
\leq & \frac{N_{0} K_{1}}{\Gamma(r)} T_{0}^{r} \zeta\left(D_{0}\right)+\frac{4 N_{1}\left(K_{1}+K_{2}\right)}{\Gamma(r+1)} T_{0}^{r} \zeta\left(D_{0}\right) \\
& +\frac{2 l_{0} N_{0} Q_{1} Q_{2} Q T^{r}}{\Gamma^{2}(r+1)} T_{0}^{r} \zeta\left(D_{0}\right)+\frac{16 l_{0} Q_{1} Q_{2} Q N_{1} T^{2 r}}{r \Gamma^{3}(r+1)} T_{0}^{r} \zeta\left(D_{0}\right) \\
\leq & \frac{Q+2 l_{0} Q_{1} Q_{2} Q T^{r}\left(N_{0}+8 N_{1} T^{r}\right)}{r \Gamma^{3}(r+1)} T_{0}^{r} \zeta\left(D_{0}\right) .
\end{aligned}
$$

On the other hand, we have from the equicontinuity and boundedness of $\mathcal{P}\left(D_{0}\right)$

$$
\zeta\left(\mathcal{P}\left(D_{0}\right)\right)=\max _{t \in W} \zeta\left(\left(\mathcal{P} D_{0}\right)(t)\right)
$$

Then, by the definition of $T_{0}$ and (12), (13), one can derive

$$
\zeta\left(D_{0}\right) \leq \zeta\left(\overline{c o}\left(\left\{z_{0}\right\} \cup \mathcal{P}\left(D_{0}\right)\right)\right) \leq \zeta\left(\mathcal{P}\left(D_{0}\right)\right)<\zeta\left(D_{0}\right),
$$

that is, $\zeta\left(D_{0}\right)=0$, which shows that $D_{0}$ is relatively compact. By Lemma 2 , we know that $\mathcal{P}$ has at least one fixed point $z \in B$, which is a mild solution to system (1) on $W$, satisfying $(\mathcal{P} z)\left(T_{0}\right)=$ $z\left(T_{0}\right)=z_{1}$. The proof is now completed.

Remark 2. (I) Compactness of the resolvent operators associated with the system (1) is unnecessary. (II) By introducing the complete space $L([-c, 0] ; E)$ and function $z_{t}$, the difficulties in the estimate of noncompactness measures caused by delay are effectively solved (Lemmas 5 and 6). Therefore, we generalize some related control results such as [20,21,43,44], etc.

\section{An Example}

To illustrate our theory, we consider the fractional integrodifferential dynamical system with delay of the form

$$
\left\{\begin{array}{l}
\frac{\partial^{\frac{3}{5}}}{\partial t^{\frac{3}{5}}} z(t, y)=\frac{\partial}{\partial y} z(t, y) \\
\quad+\frac{1}{3}\left(z(t+\theta, y)+\int_{0}^{t} \frac{(t-s)^{4}|z(s+\theta, y)|}{2+|z(s+\theta, y)|} d s\right)+\omega \mu(t, y), t \in V, y \in(0,1), \\
z(t, 0)=z(t, 1)=0, \quad t \in V, \\
z(t, y)=\psi(t, y), \quad t \in[-c, 0], y \in(0,1)
\end{array}\right.
$$

where $\psi$ is continuous and satisfies certain smoothness conditions, $\omega>0, \mu: V \times[0,1] \rightarrow$ $[0,1]$ is continuous, and $V=[0, T], \theta \in[-c, 0]$.

Let $E=U:=C([0,1])$ and let $\mathcal{A}: \mathcal{D} \subset E \rightarrow E$ given by $\mathcal{A} v=v^{\prime}$ with domain $\mathcal{D}=\left\{v \in E: v^{\prime} \in E, v(0)=v(1)=0\right\}$. So, $\mathcal{A}$ generates a semigroup $\{\mathcal{T}(t): t \geq 0\}$ on $X$, which is defined as $\mathcal{T}(t) v(s)=v(t+s)$ for $v \in E$, and $\mathcal{T}(t)$ is not a compact semigroup on $E$.

Furthermore, from the Corollary 2.4 in [45], it follows that the integral equation

$$
z(t)=\frac{1}{\Gamma(r)} \int_{0}^{t} \frac{\mathcal{A} z(s)}{(t-s)^{1-r}} d s, t \geq 0,
$$

admits an analytic resolvent operator $\mathcal{N}(t)$ on $E$.

Define

$$
\begin{aligned}
& z(t)(y)=z(t, y) \\
& { }^{C} D^{r} z(t)(y)=\frac{\partial^{\frac{3}{5}}}{\partial t^{\frac{3}{5}}} z(t, y) \\
& g\left(t, z_{t}, H_{z}(t)\right)(y)=\frac{1}{3}\left(z(t+\theta, y)+\int_{0}^{t} \frac{(t-s)^{4}|z(s+\theta, y)|}{2+|z(s+\theta, y)|} d s\right)
\end{aligned}
$$




$$
\begin{aligned}
& h\left(t, s, z_{s}\right)=\frac{(t-s)^{4}|z(s+\theta, y)|}{2+|z(s+\theta, y)|},(t, s) \in \Delta=\{(t, s) \in V \times V: s \leq t\}, \\
& x(t)(y)=\mu(t, y) .
\end{aligned}
$$

It is easy to see that $(\mathrm{H} 1)$ and $(\mathrm{H} 2)$ hold. For $y \in(0,1)$, suppose that the linear operator $\Lambda(t)$ defined as

$$
\begin{aligned}
(\Lambda(t) u)(y)= & \frac{\boldsymbol{\omega N}(t)}{\Gamma\left(\frac{3}{5}\right)} \int_{0}^{t}(t-s)^{-\frac{2}{5}} \mu(s, y) d s \\
& +\frac{\omega}{\Gamma\left(\frac{3}{5}\right)} \int_{0}^{t} \dot{\mathcal{N}}(t-s)\left(\int_{0}^{s}(s-\eta)^{-\frac{2}{5}} \mu(\eta, y) d \eta-\int_{0}^{t}(t-\eta)^{-\frac{2}{5}} \mu(\eta, y) d \eta\right) d s,
\end{aligned}
$$

satisfies the assumption (H3). In addition, simple verification can imply that (H4) holds with $k_{1}(t)=k_{2}(t)=\frac{1}{3}, t \in V$ and $l(s)=T^{4}, s \in V$. Consequently, if all the requirements of Theorem 1 are satisfied, then system (14) is controllable on $V$.

\section{Conclusions and Future Work}

Some new controllability results for a class of fractional integrodifferential dynamical systems with a delay in Banach spaces are derived in this paper by using resolvent operator theory and fixed-point theory. A new definition of controllability is introduced, and the nonlinearity is not supposed to be Lipschitz continuous compared, with most of the existing literature. A suitable delay item in a special complete space is also introduced to solve the difficulties caused by time delay. An explicit example is given to demonstrate the effectiveness of our results.

Drawing on the ideas of this paper, the controllability for a class of fractional integrodifferential dynamical inclusions with time delay and nonlocal conditions will be further studied in the future:

$$
\left\{\begin{array}{l}
D^{r} z(t) \in \mathcal{A} z(t)+g\left(t, z_{t}, H_{z}(t)\right)+\mathcal{B} x(t), \text { a.e. } t \in V:=[0, T] \\
z(t)+h(z)=\psi(t), \quad t \in[-c, 0]
\end{array}\right.
$$

where $h: C([-c, T], E) \rightarrow E$ is a given function. In common applications, the nonlocal conditions are usually described as $h(z)=\sum_{i=1}^{m} k_{i} z\left(\tau_{i}\right)$, where $k_{i}(i=1,2, \cdots, m)$ are given constants and $0<\tau_{1}<\tau_{2}<\cdots<\tau_{n} \leq T$.

Funding: This research was funded by the National Natural Science Foundation of China under grant 62073202, and a project of Shandong Province Higher Educational Science and Technology Program of China under grant J18KA233.

Institutional Review Board Statement: Not applicable.

Informed Consent Statement: Not applicable.

Data Availability Statement: Not applicable.

Acknowledgments: The authors are thankful to the editor and anonymous referees for their valuable comments and suggestions.

Conflicts of Interest: The author declares no conflict of interest.

\section{References}

1. Cheng, H.; Yuan, R. The stability of the equilibria of the Allen-Cahn equation with fractional diffusion. Appl. Anal. 2019, 98, 600-610. [CrossRef]

2. Jia, J.; Wang, H. A fast finite volume method for conservative space-time fractional diffusion equations discretized on space-time locally refined meshes. Comput. Math. Appl. 2019, 78, 1345-1356. [CrossRef]

3. Li, L.; Jiang, Z.; Yin, Z. Compact finite-difference method for 2D time-fractional convection-diffusion equation of groundwater pollution problems. Comput. Appl. Math. 2020, 39, 142. [CrossRef]

4. Mao, J.; Zhao, D. Multiple positive solutions for nonlinear fractional differential equations with integral boundary value conditions and a parameter. J. Funct. Space 2019, 2019, 2787569. [CrossRef]

5. Tian, Y.; Zhao, S. Existence of solutions for perturbed fractional equations with two competing weighted nonlinear terms. Bound. Value. Probl. 2018, 2018, 154. [CrossRef]

6. Wang, F.; Zhang, Z.; Zhou, Z. A spectral Galerkin approximation of optimal control problem governed by fractional advectiondiffusion-reaction equations. J. Comput. Appl. Math. 2021, 386, 113233. [CrossRef] 
7. Zhao, D.; Liu, Y. Twin solutions to semipositone boundary value problems for fractional differential equations with coupled integral boundary conditions. J. Nonlinear Sci. Appl. 2017, 10, 3544-3565. [CrossRef]

8. Hernández, E.; O’Regan, D.; Balachandran, K. Existence results for abstract fractional differential equations with nonlocal conditions via resolvent operators. Indag. Math. 2013, 24, 68-82. [CrossRef]

9. Ma, T.; Yan, B. The multiplicity solutions for nonlinear fractional differential equations of Riemann-Liouville type. Fract. Calc. Appl. Anal. 2018, 21, 801-818. [CrossRef]

10. Zhao, D.; Liu, Y. Positive solutions for a class of fractional differential coupled system with integral boundary value conditions. J. Nonlinear Sci. Appl. 2016, 9, 2922-2942. [CrossRef]

11. Podlubny, I. Fractional Differential Equations, Mathematics in Science and Engineering; Academic Press: New York, NY, USA, 1999.

12. Sakthivel, R.; Anthoni, S.M.; Kim, J.H. Existence and controllability result for semilinear evolution integrodifferential Systems. Math. Comput. Model. 2005, 41, 1005-1011. [CrossRef]

13. Zhao, D.; Mao, J. Positive solutions for a class of nonlinear singular fractional differential systems with Riemann-Stieltjes coupled integral boundary value conditions. Symmetry 2021, 13, 107. [CrossRef]

14. Zhao, D.; Liu, Y. Multiple positive solutions for nonlinear fractional boundary value problems. Sci. World J. 2013, $2013,473828$. [CrossRef]

15. Li, H.; Zheng, Y.; Alsaadi, F.E. Algebraic formulation and topological structure of Boolean networks with state-dependent delay. J. Comput. Appl. Math. 2019, 350, 87-97. [CrossRef]

16. Li, X.; Caraballo, T.; Rakkiyappan, R.; Han, X. On the stability of impulsive functional differential equations with infinite delays. Math. Method. Appl. Sci. 2015, 38, 3130-3140. [CrossRef]

17. Li, X.; O'Regan, D.; Akca, H. Global exponential stabilization of impulsive neural networks with unbounded continuously distributed delays. IMA J. Appl. Math. 2015, 80, 85-99. [CrossRef]

18. Wei, T.; Xie, X.; Li, X. Input-to-state stability of delayed reaction-diffusion neural networks with multiple impulses. AIMS Math. 2021, 6, 5786-5800. [CrossRef]

19. Yang, X.; Li, X.; Xi, Q.; Duan, P. Review of stability and stabilization for impulsive delayed systems. Math. Biosci. Eng. 2018, 15, 1495-1515. [CrossRef]

20. Du, J.; Jiang, W.; Pang, D.; Niazi, A.U.K. Controllability for a new class of fractional neutral integro-differential evolution equations with infinite delay and nonlocal conditions. Adv. Differ. Equ. 2017, 139, 1-22. [CrossRef]

21. Nirmala, R.J.; Balachandran, K.; Rodríguez-Germa, L.; Trujillo, J.J. Controllability of nonlinear fractional delay dynamical systems. Rep. Math. Phys. 2016, 77, 87-104. [CrossRef]

22. Tai, Z.; Lun, S. On controllability of fractional impulsive neutral infinite delay evolution integrodifferential systems in Banach spaces. Appl. Math. Lett. 2012, 25, 104-110. [CrossRef]

23. Tai, Z.; Wang, X. Controllability of fractional-order impulsive neutral functional infinite delay integrodifferential systems in Banach spaces. Appl. Math. Lett. 2009, 22, 1760-1765. [CrossRef]

24. Vijayakumar, V.; Selvakumar, A.; Murugesu, R. Controllability for a class of fractional neutral integro-differential equations with unbounded delay. Appl. Math. Comput. 2014, 232, 303-312. [CrossRef]

25. Zhao, D.; Mao, J. New controllability results of fractional nonlocal semilinear evolution systems with finite delay. Complexity 2020, 2020, 7652648. [CrossRef]

26. Hu, J.; Sui, G.; Lv, X.; Li, X. Fixed-time control of delayed neural networks with impulsive perturbations. Nonlinear Anal.-Model. 2018, 23, 904-920. [CrossRef]

27. Li, X.; Yang, X.; Huang, T. Persistence of delayed cooperative models: Impulsive control method. Appl. Math. Comput. 2019, 342, 130-146. [CrossRef]

28. Liu, Y.; Zheng, Y.; Li, H.; Alsaadi, F.E.; Ahmad, B. Control design for output tracking of delayed Boolean control networks. J. Comput. Appl. Math. 2018, 327, 188-195. [CrossRef]

29. Liu, M.; Li, S.; Li, X.; Jin, L.; Yi, C. Intelligent controllers for multirobot competitive and dynamic tracking. Complexity 2018, 2018, 4573631. [CrossRef]

30. Xu, X.; Li, H.; Li, Y.; Alsaadi, F.E. Output tracking control of Boolean control networks with impulsive effects. Math. Method. Appl. Sci. 2018, 41, 1554-1564. [CrossRef]

31. Yang, D.; Li, X.; Qiu, J. Output tracking control of delayed switched systems via state-dependent switching and dynamic output feedback. Nonlinear Anal. 2019, 32, 294-305. [CrossRef]

32. Zhao, D.; Liu, Y.; Li, X. Controllability for a class of semilinear fractional evolution systems via resolvent operators. Commun. Pur. Appl. Anal. 2019, 18, 455-478. [CrossRef]

33. Ding, X.; Li, H.; Yang, Q.; Zhou, Y.; Alsaedi, A.; Alsaadi, F.E. Stochastic stability and stabilization of n-person random evolutionary Boolean games. Appl. Math. Comput. 2017, 306, 1-12. [CrossRef]

34. Li, H.; Xu, X.; Ding, X. Finite-time stability analysis of stochastic switched boolean networks with impulsive effect. Appl. Math. Comput. 2019, 347, 557-565. [CrossRef]

35. Li, Y.; Li, H.; Li, Y. Constrained set controllability of logical control networks with state constraints and its applications. Appl. Math. Comput. 2021, 405, 126259.

36. Liang, S.; Zhao, G.; Li, H.; Ding, X. Structural stability analysis of gene regulatory networks modeled by Boolean networks. Math. Method Appl. Sci. 2019, 42, 2221-2230. [CrossRef] 
37. Yang, Q.; Li, H.; Liu, Y. Pinning control design for feedback stabilization of constrained Boolean control networks. Adv. Differ. Equ. 2016, 2016, 182. [CrossRef]

38. Zhao, G.; Liang, S.; Li, H. Stability analysis of activation-inhibition Boolean networks with stochastic function structures. Math. Method. Appl. Sci. 2020, 43, 8694-8705. [CrossRef]

39. Hernndez, E.; O’Regan, D. Controllability of Volterra-Fredholm type systems in Banach Spaces. J. Franklin. Inst. 2009, 346, 95-101. [CrossRef]

40. Hernández, E.; O'Regan, D.; Balachandran, K. On recent developments in the theory of abstract differential equations with fractional derivatives. Nonlinear Anal. 2010, 73, 3462-3471. [CrossRef]

41. Balachandran, K.; Park, J.Y. Controllability of fractional integrodifferential systems in Banach spaces. Nonlinear Anal. 2009, 3 , 363-367. [CrossRef]

42. El-Borai, M.M. Some probability densities and fundamental solutions of fractional evolution equations. Chaos Solitons Fract. 2002, 14, 433-440. [CrossRef]

43. Debbouche, A.; Baleanu, D. Controllability of fractional evolution nonlocal impulsive quasilinear delay integro-differential systems. Comput. Math. Appl. 2011, 62, 1442-1450. [CrossRef]

44. Wang, J.; Zhou, Y. Complete controllability of fractional evolution systems. Commun. Nonlinear Sci. Numer. Simulat. 2012, 17, 4346-4355. [CrossRef]

45. Prüss, J. Evolutionary Integral Equations and Applications. In Monographs in Mathematics; Birkhäuser: Basel, Switzerland, 1993; Volume 87.

46. Guo, D.; Lakshmikantham, V.; Liu, X. Nonlinear Integral Equations in Abstract Spaces; Kluwer Academic Publishers: Drive Norwell, MA, USA, 1996.

47. Liu, Y.; Regan, D.O. Controllability of impulsive functional differential systems with nonlocal conditions. Electron. J. Differ. Equ. 2013, 2013, 1-10. 